\title{
Role of TRP ion channels in physiology and pathology introduction
}

\author{
Armen Akopian ${ }^{1}$
}

Received: 8 October 2015 / Accepted: 30 October 2015 / Published online: 12 November 2015

(C) Springer-Verlag Berlin Heidelberg 2015

The transient receptor potential (TRP) channels are atypical ligand-gated cation channels that have myriad functions in the physiology and pathophysiology of higher organisms, from flies to humans. Discovery of the fly trp channel, the founding member of the TRP superfamily, stretched for almost 20 years. First, in 1969, Cosens and Manning described the behavior of mutant flies that were becoming blind under bright illumination. In 1975, Minke, Wu, and Pak characterized electrophysiology function of trp locus accounting for the CosensManning mutant. In 1989, Montell, Rubin, and colleagues cloned a candidate trp fly gene using a differential screening approach. Finally, the first mammalian homolog of fly TRP, TRPC1, was identified in the mid-1990s. The sequence of the open reading frame and basic characterization of the cloned TRP gene showed that the founding member of the TRP channel superfamily represents a new class of cation channels. TRP channels possess six transmembrane domains, S1-S6 and the pore region between S5 and S6. This structure makes TRP channels somewhat similar to voltage-gated channels; however, they lack the positively charged residues in S4. Subsequent cloning of TRP homologues revealed many sub-types that now encompass a big superfamily. Based on amino acid sequence homology, the TRP superfamily is divided into seven sub-families: TRPC, TRPM, TRPV, TRPA, TRPP, TRPML, and TRPN.

Members of the TRP channel superfamily have distinct and unique regulatory, biophysical, and pharmacological properties. There are abundant reviews in scientific journals and

Armen Akopian

Akopian@uthscsa.edu

1 The School of Dentistry, UT Health Science Center at San Antonio, 7703 Floyd Curl Dr, San Antonio, TX 78229, USA books summarizing our knowledge and perspective on these properties of TRPs and the cellular signaling pathway regulated by TRPs. The set of reviews on the "Role of TRP ion channels in physiology and pathology" in this issue of "Seminars in Immunopathology" focuses on TRP-channel function in the physiology and pathophysiology of different biological processes, from cancer to pulmonary and itch. Unique features of TRP channels allow unparalleled flexibility and critical control of $\mathrm{Ca}^{2+}$ homeostasis, regulation of intracellular $\mathrm{Ca}^{2+}$ as a pivotal second messenger, and importantly, modulation of sensory transduction pathways, including thermosensation, mechanosensation, taste perception, pheromone, and osmolarity sensing. Needless to say, the conversion of $\mathrm{Ca}^{2+}$ and especially sensory stimuli into biological responses, and understanding how these mechanisms work, provides an exciting challenge in clinically relevant pathophysiology processes. We believe that this set of reviews is timely, written for a broad audience, and will be exciting to read. The reviews follow a "molecule to disease" presentation style, and use a "bench to bedside" methodological approach.

An average of more than 600 papers has appeared annually on the subject of TRPs since 1995. It is impossible to deal with all aspects of "TRPs in pathophysiology" in a single issue of the journal. Nevertheless, we have attempted to cover a wide variety of the topics on "TRPs in pathophysiology" with an emphasis on cellular and molecular mechanisms, which are written by the world leaders in their corresponding fields. Thus, this issue reviews functions of TRPV1-4, TRPM8, and TRPA1 as transducers for signals from thermal, chemical, and mechanical noxious stimuli and their participation in controlling the generation and development of pathological pain. Besides the pain pathways, TRP channels are able to regulate function of specialized subset "itch-sensing" nerves. "TRP channel and itch" provides an overview of the current understandings on functioning of primary itch-sensing population, 
and involvement of TRP channels in control of acute as well as chronic itch mechanisms. Interaction between Toll-like receptors (TLR) and TRP is of especial interest in this review. The capability of certain TRP channels to act as chemical sensors and control $\mathrm{Ca}^{2+}$ influx in nerves is explored in relevant gastrointestinal disorders via mechanisms controlling the interaction between afferent nerves and gastrointestinal tract structures such as the esophagus, stomach, intestine, and colon. The review on "TRPs and cancer" gives an exciting overview on TRP channel involvement in the regulation of cell fate via the regulation of $\mathrm{Ca}^{2+}$ homeostasis in various transformed tissues. The newly discovered and intriguing TRPmediated modulation of renal function along the nephron involves multiple downstream signaling pathways. These include indirect activation of TRPC6 by mechanical stimuli and the TRPV4-TRPP2 complex by liquid flow, as well as functional interaction between angiotensin receptor and TRPs. Function of certain TRP channels as environmental sensors makes them important players in regulation of cardiovascular and pulmonary systems. One of the main questions in this research direction is whether neuronal or non-neuronal TRPs control these systems, and what functional relationship there is, if any, between the neuronal and non-neuronal TRPs. Another interesting research direction is on the involvement of TRPs in metabolism. Recent published data showed that TRPV1 and TRPA1 agonists have beneficial effects on whole body metabolism including glucose homeostasis. Thus, the "potential therapeutic value of TRPV1 and TRPA1 in diabetes mellitus and obesity" provides an overview on possible TRP channel mechanisms that may control metabolism. Interestingly, some TRP antagonists have negative effects on thermogenesis, leading to hyperthermia and preventing their use clinically. An important and relevant review summarizes the function of TRPs in immune cells, specifically T cells. Recent publications in this research area are critical, since it was shown that although the magnitude of TRP response in immune cells is small compared to neuronal TRPs, they still have a vital role in the regulation of the pathophysiological process. Finally, another topic discussed here is the potential role of TRPs in mediating the effects of diesel exhaust on pulmonary function. This is possibly the least-developed area of research, but is just as critically important as the research in other TRP fields. New technologies are appearing on the market for a new generation of fuels including different types of clean diesels. In such circumstances, no reliable legislation can be adopted by governments without first evaluating the health benefits and drawbacks of this new generation of fuels.

In conclusion, it is hoped that bringing together all these reviews written by the field's leaders will produce a timely and gratifying read. One that will promote collaborations among different disciplines, inspiring new ideas that will result in a deeper understanding of TRPs and the myriad physiological and pathological processes they have a role in as well as the development of new therapeutic approaches. 